\title{
AN ANALOGUE OF THE MULTINOMIAL THEOREM
}

\author{
T. V. Na rayana
}

(received July 17, 1961)

1. Let $x_{i}, y_{i}(i=1,2, \ldots, t)$ and $n$ be non-negative integers. A function $\left(n ; x_{1}, \ldots, x_{t}\right)$ may be defined recursively as follows: let $(0 ; 0, \ldots, 0)=1$ and

$$
\left(n ; x_{1}, \ldots, x_{t}\right)=\left\{\begin{array}{l}
0 \text { if } \sum_{i=1}^{t} x_{i}>n \\
\sum_{y_{1}=0}^{x_{1}} \cdots \sum_{y_{t}=0}^{x_{t}}\left(n-1 ; y_{1}, \ldots, y_{t}\right) \text { othe rwise. }
\end{array}\right.
$$

If $\Delta_{n}$ denotes the $t \times t$ determinant given by

$$
\begin{aligned}
\Delta_{n}\left(x_{1}, \ldots, x_{t}\right)=\Delta_{n} & =\left|a_{r s}\right|_{r, s=1, \ldots, t} a_{r s}=\left\{\begin{array}{l}
-x_{r} \text { if } r \neq s \\
n-x_{r} \text { if } r=s
\end{array}\right. \\
& =n\left[1-\frac{1}{n} \sum_{i=1}^{t} x_{i}\right],
\end{aligned}
$$

then it is easy to verify that, when $\sum_{i=1}^{t} x_{i} \leqq n$,

$$
\left(n ; x_{1}, \ldots, x_{t}\right)=\Delta_{n+1} \prod_{i=1}^{t}\left(n+1+x_{i}\right)^{-1}\left(\begin{array}{c}
n+1+x_{i} \\
x_{i}
\end{array}\right)
$$

Canad. Math. Bull. vol. 5, no. 1, January 1962. 
where the last symbol denotes a binomial coefficient. Interest in the relation (4) a rose in a study of simple sampling plans of size $n$ and it includes, as special cases, some known ballot theorems in probability $[1,66, \underline{5}, \underline{6}]$. In this note* we confine ourselves to the case $t=3$ (apart from a verification of (4) in 62); our purpose being to exhibit its connection with a certain set $T(n)$ of vectors forming a distributive lattice, partially ordered by a relation of domination [cf. $2, \underline{3}]$. If $n \geqq 1$, and $a_{1}, \ldots, a_{n}$ are non-negative integers, $T(n)$ consists of the vectors $A_{n}=\left(a_{1}, \ldots, a_{n}\right)$ which satisfy

$$
\begin{aligned}
& \text { (i) } 0 \leqq a_{1} \leqq a_{2} \leqq \cdots \leq a_{n}, \\
& \text { (ii) } \quad a_{i} \leqq 3 i(i=1,2, \ldots, n) .
\end{aligned}
$$

$A_{n}$ is said to dominate $B_{n}$ if, and only if, $a_{i} \geqq b_{i}(i=1,2, \ldots, n)$ and both $A_{n}$ and $B_{n}$ are in $T(n)$. We introduce certain subsets $T(n, r)$ and $S_{r}\left(n ; x_{1}, x_{2}, x_{3}\right)$ of $T(n)$, where $r=0,1, \ldots, n$, $x_{1}+x_{2}+x_{3}=r$. Let

$$
\begin{aligned}
& T(n, 0)=\{(0, \ldots, 0)\}, T(n, n)=\left\{A_{n} \mid a_{1}>0\right\} \\
& T(n, r)=\left\{A_{n} \mid a_{1}=\ldots a_{n-r}=0, a_{n-r+1}>0\right\}, r=1, \ldots, n-1 .
\end{aligned}
$$

Thus $T(n, r), 0 \leqq r \leqq n$ form a partition of $T(n)$. Also, if $A_{n} \in T(n, r)$, then it has exactly $r$ positive components $a_{n-r+1}, \ldots, a_{n}$, by (1). Of these $r$ components, let $x_{i}$ of them be $\equiv i-1(\bmod 3)$ so that

$$
x_{1}+x_{2}+x_{3}=r
$$

For fixed $x_{1} \geq 0, x_{2} \geq 0, x_{3} \geq 0$ with $x_{1}+x_{2}+x_{3}=r$, let $S_{r}\left(n ; x_{1}, x_{2}, x_{3}\right)$ denote the set of all $A_{n}$ in $T(n, r)$ with this

*The case $t=2$ was treated in [ 4 ] 
property. Since the number of solutions of $x_{1}+x_{2}+x_{3}=r$ in non-negative integers is $\left(\begin{array}{c}r+2 \\ 2\end{array}\right)$, we see that $T(n, r)$ is partitioned into $\left(\begin{array}{c}r+2 \\ 2\end{array}\right)$ sets $S_{r}\left(n ; x_{1}, x_{2}, x_{3}\right)$.

Let $\left[n, r ; x_{1}, x_{2}, x_{3}\right]$ denote the number of vectors in $S_{r}\left(n ; x_{1}, x_{2}, x_{3}\right)$. Then we shall prove, in $\$ 3$, that it is independent of $r$ and, moreover,

$$
\left[n, r ; x_{1}, x_{2}, x_{3}\right]=\left(n ; x_{1}, x_{2}, x_{3}\right)
$$

where the function on the right is given in (4), with $t=3$.

We remark that the set $T(n)$ and its relation of domination have a simple geometrical interpretation in the plane, which indicates a connection with ballot theorems [cf. 1, 5 ]. Consider the triangle $\Delta$,

$$
0 \leqq 3 y \leqq x \leqq 3 n+4
$$

and let $P(n)$ be the path from $O(0,0)$ to $P(3 n+4, n)$ inside $\Delta$ of the form

$$
\left\{O P_{0} P_{0}^{\prime} P_{1} P_{1}^{\prime} \cdots P_{n-1} P_{n-1}^{\prime} P\right\}
$$

where $P_{n-j}=\left(3 n+4-a_{j}, n-j\right), P_{n-j}^{\prime}=P_{n-j}+(0,1)$.

Clearly any vector of $T(n)$ gives a path $P(n)$ of this type and conversely. Also, the relation of domination for $T(n)$ means that if $A_{n}$ dominates $B_{n}$ then the path corresponding to $B_{n}$ is never above that of $A_{n}$.

I wish to thank the referees for preparing the final draft of my paper, and their helpful suggestions.

2. Proof of (4). It is clearly true for $n=0,1$. Suppose then that (4) holds for $n=k-1(k \geqq 2)$ and $\Sigma x_{i} \leqq k-1$, we will prove that (4) holds for $n=k$ and $\Sigma x_{i} \leqq k$. Now, by (1), 
$\left(k ; x_{1}, \ldots, x_{t}\right)=\sum_{y_{1}=0}^{x_{1}} \cdots \sum_{y_{t}=0}^{x_{t}}\left(k-1 ; y_{1}, \ldots, y_{t}\right)$.

Since $\Sigma x_{i} \leqq k$, we have $\Sigma y_{i}<$ k unless $y_{1}=x_{1}, \ldots, y_{t}=x_{t}$ and $x_{1}+\ldots+x_{t}=k$. This is a single term and, by (1), has value 0 . Hence we can suppose $\Sigma x_{i} \leqq k-1$, in which case we can apply (4) to obtain

$$
\left(k ; x_{1}, \ldots, x_{t}\right)=\Sigma \ldots \Sigma \prod_{i=1}^{t}\left(k+y_{i}\right)^{-1}\left(y_{i}^{k+y_{i}}\right) \Delta_{k}\left(y_{1}, \ldots, y_{i}\right) .
$$

Taking the factor $\left(k+y_{i}\right)^{-1}\left(\begin{array}{c}k+y_{i} \\ y_{i}\end{array}\right)$ into the $i^{\text {th }}$

column of $\Delta$, we can effect the sum with respect to $y_{i}$ by adding the $t$ determinants, which differ only in the $i^{\text {th }}$ column, in the usual way. Repeating for $i=1,2, \ldots, t$, we see that the sum on the right is itself a determinant, say $\left|d_{r s}\right|$, where

$$
d_{r S}=\left\{\begin{array}{c}
-\sum_{y_{r}=0}^{x_{r}} \frac{y_{r}}{k+y_{r}}\left(\begin{array}{c}
k+y_{r} \\
y_{r}
\end{array}\right) \text { if } r \neq s \\
\sum_{y_{r}=0}^{x_{r}} \frac{k-y_{r}}{k+y_{r}}\left(\begin{array}{c}
k+y_{r} \\
y_{r}
\end{array}\right) \text { if } r=s .
\end{array}\right.
$$

Since

$$
\left(\begin{array}{l}
k+j \\
j-1
\end{array}\right)=\sum_{x=1}^{j}\left(\begin{array}{c}
k+j-x \\
j-x
\end{array}\right)
$$

it follows that

$$
\left|d_{r S}\right|=\prod_{i=1}^{t}\left(k+1+x_{i}\right)^{-1}\left(k+\frac{1}{x_{i}}+x_{i}\right) \Delta_{k+1}\left(x_{1}, \ldots, x_{t}\right) .
$$


3. Theorem: $\left[n, r ; x_{1}, x_{2}, x_{3}\right]=\left(n ; x_{1}, x_{2}, x_{3}\right)$.

Proof. Since the theorem holds for $n=0,1$, it is sufficient to prove that

$$
\left[n, r ; x_{1}, x_{2}, x_{3}\right]=\sum_{y_{1}=0}^{x_{1}} \sum_{y_{2}=0}^{x_{2}} \sum_{y_{3}=0}^{x_{3}}\left(n-1 ; y_{1}, y_{2}, y_{3}\right)
$$

for $n \geqq 2$.

Let $r$ be any fixed integer satisfying $0 \leqq r \leqq n$. Then this may be effected by setting up a $(1,1)$ correspondence between the sets $S_{r}\left(n ; x_{1}, x_{2}, x_{3}\right)$ and

$$
S^{*}\left(n-1 ; x_{1}, x_{2}, x_{3}\right)=y_{1}, y_{2}, y_{3} S_{t}\left(n-1 ; y_{1}, y_{2}, y_{3}\right) \text {, }
$$

where $0 \leqq y_{i} \leqq x_{i}(i=1,2,3)$ and $t$ denotes $y_{1}+y_{2}+y_{3}$. Let

$$
\begin{aligned}
& S_{r}^{\circ}\left(n ; x_{1}, x_{2}, x_{3}\right)=\left\{A_{n} \mid A_{n} \in T(n, r), a_{i}>3,(i=1,2, \ldots, n)\right\} \\
& S_{r}^{n}\left(n ; x_{1}, x_{2}, x_{3}\right)=\left\{A_{n} \mid A_{n} \in T(n, r), a_{i} \leqq 3,(i=1,2, \ldots, n)\right\} \\
& S_{r}^{k}\left(n ; x_{1}, x_{2}, x_{3}\right)=\left\{A_{n} \mid A_{n} \in T(n, r), a_{k} \leqq 3, a_{k+1}>3\right\}, \\
& k=1,2, \ldots, n-1 .
\end{aligned}
$$

Then $s_{r}^{k}\left(n ; x_{1}, x_{2}, x_{3}\right), k=0,1, \ldots, n$ form a partition of $S_{r}\left(n ; x_{1}, x_{2}, x_{3}\right)$. Consider the mapping $P_{i}$ of $S_{r}^{1}\left(n ; x_{1}, x_{2}, x_{3}\right)$

into the set $S=\left\{P_{i}\left(A_{n}\right) \mid A_{n} \in S_{r}^{i}\left(n ; x_{1}, x_{2}, x_{3}\right)\right\}$, where $P_{i}\left(A_{n}\right)$ is obtained from $A_{n}$ by the following three operations

(a) suppress the first element of $A_{n}$ '

(b) replace the next $i-1$ elements of $A_{n}$ by 0 ,

(c) subtract 3 from the last $n$ - i elements of $A_{n}$. 
Thus $P_{i}\left(A_{n}\right)=A_{n-1}$ say, where $A_{n-1} \in S_{n-i}^{j}$ for some $j \geqq i-1$. In fact, if $x_{k}^{\prime}$ of the elements of $P_{i}\left(A_{n}\right)$ are $\equiv k-1(\bmod 3)$, we have

$$
P_{i}\left(A_{n}\right) \in S_{n-i}^{j}\left(n-1, x_{1}^{\prime}, x_{2}^{\prime}, x_{3}^{\prime}\right)
$$

We first show that $P_{i}$ is a $(1,1)$ mapping of $s_{r}^{i}\left(n ; x_{1}, x_{2}, x_{3}\right)$ into S. Suppose then that $P_{i}\left(A_{n}\right)=P_{i}\left(B_{n}\right)$. This means that $P_{i}\left(A_{n}\right)$ and $P_{i}\left(B_{n}\right)$ a re both elements of some $S_{n-i}^{j}\left(n-1 ; x_{1}^{\prime}, x_{2}^{\prime}, x_{3}{ }^{\prime}\right)$ and, in particular, that the last $n-i$ elements a re identical. Let $A_{n}=\left(a_{1}, \ldots, a_{i}, a_{i+1}, \ldots a_{n}\right), B_{n}=\left(b_{1}, \ldots, b_{i}, a_{i+1}, \ldots, a_{n}\right)$, since the must also have identical elements in the last $n-i$ places by $(c)$. Since $r>n-i$, we consider the elements

$$
\begin{aligned}
& a_{n-r+1} \cdots a_{i}, \\
& b_{n-r+1} \cdots b_{i} .
\end{aligned}
$$

By (5) and (6) we see that they are all positive and $\leqq 3$. Let $x_{j}$ of the se $a^{\prime} s$ be $\equiv j-1(\bmod 3)$. Since $a_{k}=b_{k}(k=i+1, \ldots, n)$, we see that $y_{j}$ of these $b^{\prime} s$ are $\equiv j-1(\bmod 3)$. Moreover, they are all $\leq 3$. Hence

either $y_{1} \neq 0$ and $a_{n-r+1}=\ldots=a_{i}=b_{n-r+1}=\ldots=b_{i}=3$ or $y_{1}=0$ and $\left\{\begin{array}{l}a_{n-r+1}=\ldots=a_{n-r+y_{2}}=1 ; a_{n-r+y_{2}+1}=\ldots=a_{i}=2 \\ b_{n-r+1}=\ldots=b_{n-r+y_{2}}=1 ; b_{n-r+y_{2}+1}=\ldots \Rightarrow_{i}=2 .\end{array}\right.$

Hence $A_{n}=B_{n}$. Consider now the mapping $P$ of $S_{r}\left(n ; x_{1}, x_{2}, x_{3}\right)$ into $S^{*}\left(n-1 ; x_{1}, x_{2}, x_{3}\right)$ where $P\left(A_{n}\right)=P_{i}\left(A_{n}\right)$ whenever $A_{n} \in S_{r}^{i}\left(n ; x_{1}, x_{2}, x_{3}\right),(i=0,1, \ldots n)$. Clearly $P_{i}\left(A_{n}\right) \in S^{*}$, since $P_{i}\left(A_{n}\right) \in S_{n-i}^{j}\left(n-1 ; y_{1}, y_{2}, y_{3}\right)$ for some $j \geqq i-1$ and $0 \leqq y_{i} \leqq x_{i}$, 
$(i=1,2,3)$ by $(a),(b),(c)$. We note also that $P$ is $(1,1)$, for supposing $A_{n} \in S_{r}^{i}, B_{n} \in S_{r}^{k}$, we have

$$
P\left(A_{n}\right) \in S_{n-i}^{j}(j \geqq i-1), P\left(B_{n}\right) \in s_{n-k}^{l}(\ell \geqq k-1),
$$

and then, $P\left(A_{n}\right)=P\left(B_{n}\right)$ implies that $n-i=n-k$ or $i=k$ and $\mathrm{j}=\ell$ and so $P=P_{i}$.

Finally, we show that $P$ is a $(1,1)$ map onto $S^{*}$. Suppose then that $A_{n-1} \in S_{n-j}^{k}\left(n-1 ; y_{1}, y_{2}, y_{3}\right)$, where $0 \leqq y_{i} \leqq x_{i}$. Write $A_{n-1}=\left(0, \ldots 0, a_{j+1}^{\prime}, \ldots, a_{n}^{\prime}\right)$ and consider any $x_{n} \in s_{r}^{j}\left(n ; x_{1}, x_{2}, x_{3}\right)$, where

$$
x_{n}=\left(a_{1}, \ldots, a_{j}, a^{\prime}{ }_{j+1}, \ldots, a^{\prime}{ }_{n}\right) .
$$

Observe that $a_{1}=\ldots=a_{n-r}=0$, and we may choose

$$
\begin{aligned}
& a_{n-r+1}=\ldots=a_{n-r+\left(x_{2}-y_{2}\right)}=1, \\
& a_{(n-r)}+\left(x_{2}-y_{2}\right)+1=\ldots=a_{(n-r)}+\left(x_{2}-y_{2}\right)+\left(x_{3}-y_{3}\right)=2,
\end{aligned}
$$$$
{ }^{a}(n-r)+\left(x_{3}-y_{3}\right)+1=\ldots=a_{j}=3 \text {, }
$$$$
\text { since }(n-r)+\left(x_{2}-y_{2}\right)+\left(x_{3}-y_{3}\right)+\left(x_{1}-y_{1}\right)=j \text {. }
$$

Thus, for this particular $x_{n}$, there are exactly $n-r$ zero elements, $\left(x_{2}-y_{2}\right)+y_{2}$ elements $\equiv 1(\bmod 3),\left(x_{3}-y_{3}\right)+y_{3}$ elements $\equiv 2(\bmod 3)$ and $\left(x_{1}-y_{1}\right)+y_{1}$ elements $\equiv 0(\bmod 3)$. This concludes the proof.

4. It is evident that the method of $\S 3$ is rather more general, in that we could, for example, consider sets of vectors $\left(a_{1}, \ldots, a_{n}\right)$ satisfying

$$
\begin{aligned}
& \text { (i) } 1 \leqq a_{1} \leqq a_{2} \leqq \cdots \leqq a_{n} \\
& \text { (ii) } \quad a_{i} \leqq \lambda i \quad(i=1, \ldots, n) .
\end{aligned}
$$


Even the condition (ii)' could be weakened to read $a_{i} \leqq k K_{i}$ ' where $0<\mathrm{K}_{1}<\ldots<\mathrm{K}_{\mathrm{n}}$. But such questions do not have such immediate importance in their applications to statistics.

\section{REFERENCES}

1. W. Feller, An introduction to probability theory and its applications, Vol. 1 (New York 1957).

2. T.V. Narayana, Sur les treillis formés par les partitions $\mathrm{d}^{\prime}$ un entier; leurs applications à la théorie des probabilitiés. CR., vol. 240, pp. 1188-89 (1955).

3. T.V. Narayana and G. E. Fulton, A note on the compositions of an integer. Can. Math. BuIl. Vol. 1, No. 3, pp. 169-173, Sept. 1958.

4. B. Brainerd and T. V. Narayana, A Note on Simple Binomial Sampling Plans. Annals of Math. Statistics, 32, (1961), 906-8.

5,6 T.V. Narayana and S. G. Mohanty, Some properties of compositions and their applications to probability and statistics I, II (submitted to Biometrische Zeitschrift).

University of Alberta

and National Institute of Arthritis and Metabolic Diseases. 